\title{
Traumatherapie in der psychotherapeutischen Praxis
}

\author{
Herausforderungen und Möglichkeiten bei der Arbeit mit komplex \\ traumatisierten KlientInnen
}

\author{
Martina Bernhaupt-Hopfner · Theresia Kosicek
}

Eingegangen: 25. Januar 2017 / Angenommen: 27. März 2017 / Online publiziert: 10. Mai 2017

(c) Der/die Autor(en) 2017. Dieser Artikel ist eine Open-Access-Publikation.

Zusammenfassung Aus der Erfahrung als niedergelassene Psychotrauma-Therapeutinnen beschreiben die Autorinnen Herausforderungen in der therapeutischen Arbeit mit komplex traumatisierten KlientInnen. Dabei werden sowohl Faktoren der Therapie selbst (Beziehung, Kontinuität, Support u. a.m.), als auch Faktoren der Kontexte (Gesundheitssystem, Finanzierungssysteme, Helfende u.a.m.) betrachtet. Zusätzlich wird auf Herausforderungen in der professionellen Rolle (Vernetzung, Supervision, Stresstoleranz etc.) eingegangen. Es werden Problematiken in Ausbildungs- und Versorgungssystemen diskutiert und skizziert, wie diesen begegnet werden kann. Empfehlungen für die Trauma-therapeutische Praxis schließen den Beitrag ab.

Schlüsselwörter Psychotraumatherapie - Freie psychotherapeutische Praxis - Psychotherapie - Psychotherapieausbildung · Psychotherapiefinanzierung

\section{Psycho-trauma-therapy in private practice Challenges and opportunities working therapeutically with complex trauma}

Summary The authors describe challenges in working therapeutically with trauma patients, especially patients with complex trauma, in private practice. The focuses are factors of therapy itself (therapeutic relationship, continuity, support ...) as well as the con-

Diese Arbeit ist Teil des Leitthemas „Traumatherapie in der Praxis“.

M. Bernhaupt-Hopfner ( $\square)$

Schüttelstraße 43/6, 1020 Wien, Österreich

praxis@bernhaupt-hopfner.at

T. Kosicek $(\square)$

Gussenbauergasse 1/19, 1090 Wien, Österreich

praxis@theresia-kosicek.at texts of therapy (healthcare system, funding systems, multidisciplinarity ...) and role related aspects (networking, supervision, stress tolerance ...). Room for improvement is discussed regarding therapeutic education as well as healthcare system. Recommendations for how to face and overcome these challenges complete the article.

Keywords Psychotraumatherapy • Private psychotherapeutic practice $\cdot$ Psychotherapy $\cdot$ Psychotherapeutic education · Funding of psychotherapy

\section{Einleitung}

Hat sich die Forschung bisher mit dem Thema ,traumatherapeutische Arbeit mit komplex traumatisierten Menschen in der freien Psychotherapie-Praxis“ beschäftigt? Literatur findet man eigentlich nur zur umfassenden Forschung zum Wie von Therapie, zu entsprechenden Therapie-Manualen, auch Studien zum Outcome bestimmter Therapieverfahren in definierten Settings (z. B. Therapie-Units) mit definierten Zielgruppen (z. B. nach einem Unfall Versehrte). Auch die (neuropsychologische) Forschung ermöglicht ein immer fundierteres Verständnis von Phänomenen wie beispielsweise struktureller Dissoziation (Nijenhuis 2016). Praxeologische Forschung, wie Traumatherapie in der freien Praxis gelingen kann und gelingt, scheint jedoch bislang kein großes Thema in der Forschung gewesen zu sein.

Es werden im Folgenden die Herausforderungen bei traumatherapeutischem Arbeiten in der niedergelassenen therapeutischen Praxis mit komplex traumatisierten KlientInnen eingehender beschrieben, strukturiert und im Anschluss daran Lösungsmöglichkeiten aufgezeigt.

Die praktischen Problemstellungen, die in der Arbeit mit traumatherapeutischem Schwerpunkt häufig 
auftreten, ergeben sich zum einen aus der speziellen Dynamik der Arbeit mit komplex traumatisierten KlientInnen. Zum anderen sind es Faktoren des Gesundheitssystems, die die Arbeit mit diesen KlientInnen massiv erschweren können. Wir wollen nachfolgend neben einem Fokus auf diese Dynamiken und Faktoren auch noch einen Blick auf den Ausbildungsstand in Sachen Traumatherapie richten.

Wir beschreiben in unserem Beitrag die Arbeit v. a. mit jenen KlientInnen, die nach gängiger Fachliteratur und Diagnostik (Interview zur komplexen posttraumatischen Belastungsstörung, IkPTBS (Sack 2001)) als „komplex traumatisiert“ gelten. Der Begriff „komplex traumatisiert“ wurde erstmals von der amerikanischen Traumaforscherin Judith Herman vorgeschlagen, um die vielfältigen Symptome des Störungsbildes als Folge chronischer Traumatisierung zu erfassen (Herman 1992; Sack 2001). Die Betroffenen haben eine Fülle von Beschwerden und enorme Symptomatik, die Therapie dauert nicht selten viele Jahre. Die Diagnose „komplex traumatisiert“ ist gerechtfertigt, wenn Menschen neben dem Erlebnis traumatischer Erfahrung(en) auch Veränderungen in der Affektregulation und Impulskontrolle, in der Selbstwahrnehmung, in Aufmerksamkeit und Bewusstsein, Beeinträchtigungen in der Wahrnehmung und Gestaltung der Beziehung zu anderen Menschen, Veränderungen persönlicher Glaubens- und Wertvorstellungen sowie somatische Symptome aufweisen. Oft besteht ein Spektrum an dissoziativen Phänomenen bis hin zur strukturellen Dissoziation, häufig bestehen vielfältige Komorbiditäten im Bereich Somatisierung, Zwang, Angst, Depression, Sucht.

In der Literatur werden für diesen Symptomkomplex verschiedene Formulierungen verwendet, z. B. komplexe PTBS, andauernde Persönlichkeitsveränderung nach Extrembelastung (ICD 10 (WHO 1994): F62.0), Disorders of Extreme Stress Not Otherwise Specified (DESNOS), Entwicklungstrauma-Störung (Köllner und Maercker 2011). In der derzeitigen Diskussion um die ICD-11 wird von Trauma-ExpertInnen eine Ergänzung um die Diagnose „komplexe PTBS“ angestrebt. Diese würde die irreführenden und vielfältigen Einzeldiagnosen ersetzen, die bei Menschen mit komplexer PTBS andernfalls zu vergeben sind, jedoch aus fachlicher Sicht nicht den Behandlungsnotwendigkeiten bei komplexer PTBS gerecht werden.

Zusätzlich gibt es in der freien Praxis häufig KlientInnen, die nicht in allen Items das ausgeprägte Vollbild der komplexen PTBS zeigen, jedoch mehr - und manchmal weit mehr - als nur eine klassische PTBS. Es sind auch bei diesen KlientInnen in unterschiedlichem Ausmaß zur PTBS bzw. Traumatisierung weitere Komorbiditäten wie beispielsweise Depression, Sucht, Zwang oder dissoziative Phänomene vorhanden, die Behandlung in freier Praxis gestaltet sich dem entsprechend herausfordernd. Auf diese Herausforderungen wollen wir nachfolgend einen genaueren Blick werfen.
Herausforderungen bei der Arbeit mit komplex traumatisierten Klientlnnen in der freien psychotherapeutischen Praxis

\section{Trauma-spezifische Qualifikation}

Für die Behandlung einer PTBS (und folglich auch assoziierter Folgestörungen, Anm. der Autorinnen) lautet die Empfehlung der nationalen Versorgungsleitlinie S3 zur PTBS in Deutschland, welche von den führenden Trauma-Fachgesellschaften Deutschlands unter Einbezug hochqualifizierter ExpertInnen erstellt wurde: „Eine traumaadaptierte Psychotherapie soll jedem Patienten mit PTBS angeboten werden“ und „Die behandelnden Psychotherapeutinnen sollen über eine traumatherapeutische Qualifikation verfügen" (Flatten et al. 2011).

Fachwissen aus dem Bereich der Psychotraumatologie wird jedoch in den meisten Therapieausbildungen, jedenfalls in Österreich, trotz langjähriger und kostenintensiver Ausbildung bisher kaum oder nicht vermittelt. Wie Silke Birgitta Gahleitner (2010) es formulierte: „Kenntnisse zur Psychotraumatologie gehören nach wie vor nicht zu den regulären Ausbildungsinhalten für PsychotherapeutInnen und BeraterInnen, egal welcher Grundlagendisziplin“.

Eine Recherche auf den Homepages der 28 (von insgesamt 39 vom Bundesministerium anerkannten) Ausbildungsvereinen, die Mitglieder des ÖBVP sind $^{1}$, zeigt ein entsprechendes Bild. Eine Suche nach dem Stichwort Trauma in den jeweils angegebenen Ausbildungsinhalten oder, soweit vorhanden, in den aktuellen Ausbildungsseminaren ergibt bei fünf (!) der 28 Anbieter in den Fachspezifika (mind.) ein Seminar, das dezidiert „Trauma“ zum Inhalt macht (Angaben soweit aufgrund der komplexen unterschiedlichen Darstellung ersichtlich). In keiner der recherchierten 28 Ausbildungseinrichtungen wird Trauma als Schwerpunkt in der fachspezifischen Ausbildung hervorgehoben, in einigen wenigen Ausbildungen werden spezifische Fortbildungen für PsychotherapeutInnen angeboten (jedoch diese überwiegend nicht im Rahmen der Ausbildung, sondern zusätzlich bzw. nach Abschluss der Ausbildung). Die Vermittlung von therapeutischem Knowhow aus einem sehr wesentlichen und grundlegenden Forschungsgebiet der letzten beiden Jahrzehnte findet also in unseren fachspezifischen Therapie-Ausbildungen weitgehend nicht statt. Fundiertes therapeutisches Fachwissen in Psychotraumatologie und Psychotraumatherapie kann derzeit überwiegend nur im Rahmen von spezifischen Fort- bzw. umfangreichen Weiterbildungen nach Ende der Ausbildung (bzw. teilweise schon im Status i. A. u. S.) erworben werden.

\footnotetext{
1 Stand 21.01.2017, https://www.psychotherapie.at/ psychotherapeutinnen/bildung/fachspezifika.
} 
Eine in Fachkreisen sehr anerkannte curriculare Weiterbildung ist beispielsweise das Curriculum „Spezielle Psychotraumatherapie (DeGPT)“ in Deutschland. Die Deutschsprachige Gesellschaft für Psychotraumatologie (DeGPT) hat Standards zur Qualifikation für die spezialisierte psychotherapeutische Behandlung von Erwachsenen mit Traumafolgestörungen definiert. Durch den Nachweis einer curricularen Weiterbildung nach diesem Standard an einem zertifizierten Weiterbildungsinstitut können deutsche PsychotherapeutInnen die Qualifikation „Spezielle Psychotraumatherapie (DeGPT)“ erwerben. Auch in Österreich gibt es mittlerweile einige Anbieter von Fortbildungen im Bereich Psychotraumatologie für PsychotherapeutInnen sowie andere Berufsgruppen. Einige Institute bieten eine Weiterbildung entsprechend den Anforderungen des Bundesministeriums für curriculare Weiterbildung für PsychotherapeutInnen in Österreich an. Auch die Ausbildung nach dem deutschen DeGPT-Standard kann in Österreich erworben werden; zusätzlich werden vielfältige Spezialseminare für methodische Vertiefung im Rahmen der Behandlung von Traumafolgestörungen angeboten.

Der Ausbildungsstand niedergelassener PsychotherapeutInnen in Österreich im Fachgebiet Psychotraumatologie/Traumatherapie kann also von entweder gar keiner spezifischen Ausbildung oder dem Besuch eines oder weniger Seminare bis hin zur Absolvierung eines Curriculums und etlicher weiterer Spezialseminare reichen.

Diesen Unterschieden entsprechend stellt bereits die Nachvollziehbarkeit der Ausbildung des/der gewählten PsychotherapeutIn eine große Herausforderung bzw. Hürde für KlientInnen dar, falls diese gezielt traumatherapeutisch ausgebildete KollegInnen suchen. So konnte z. B. der VAP (Verein für Ambulante Psychotherapie) auf unsere Anfrage keine Auskunft über die Anzahl der Vereinsmitglieder mit Traumaspezifischer Weiterbildung geben. Die Website des wlp (Wiener Landesverband für Psychotherapie) listet für Wien $316^{2}$ KollegInnen mit einem Schwerpunkt Trauma/Belastungsstörung auf. Die Plattform bestHELP.at führt österreichweit $1381^{3}$ PsychotherapeutInnen an, die das Arbeits-Fachgebiet „Trauma/ Belastungsstörungen“ angeben. Psychnet, das Online-Informationssystem für psychologische Dienstleistungen, listet insgesamt 1896 PsychologInnen mit dem Arbeits-Schwerpunkt „Trauma“ auf. ${ }^{4}$ All diese Informationen beruhen jedoch auf Selbstangabe.

Es existieren jedoch auch Websites der Interessensvertretungen bzw. Fachgesellschaften für Traumatherapie wie z. B. die des ÖNT (Österreichisches Netzwerk

\footnotetext{
2 Gesehen 16.01.2017, http://psychotherapie-wlp.at/

informationen/psychotherapeutinnen-suche.

3 Gesehen 16.01.2017, http://www.besthelp.at/.

4 Gesehen 19.10.2016, https://www.psychnet.at/.
}

für Traumatherapie) oder des EMDR-Netzwerk Österreich (Fachgesellschaft für spezifische Traumatherapie), auf der nur jene KollegInnen gelistet werden, die nachweislich durch umfangreiche Fortbildungen bzw. Weiterbildung Fachkenntnisse und entsprechende Arbeitstechniken bis hin zu o. g. international anerkannten Zertifizierungen in Psychotraumatologie erworben haben. Auf der Website des ÖNT finden sich 122 Personen $^{5}$, auf der Website des EMDR-Netzwerks Österreich sind es 986, die die Anforderungen für die Nennungen erfüllen und hier gelistet sind.

Fazit: Es ergibt sich in der Fülle der Informationsquellen ein sehr unterschiedliches und unübersichtliches Bild darüber, wer über welche Expertise in der Traumatherapie verfügt.

\section{Besondere Anforderungen in der Diagnostik}

Eine grundlegende Herausforderung in der therapeutischen Praxis stellt bereits das Erkennen einer komplexeren traumabedingten Folgestörung dar. Die korrekte Diagnose von dissoziativen Symptomen beispielsweise ist selbst unter Fachleuten weder selbstverständlich noch weit verbreitet, wie eine Studie aus Deutschland zeigt. In dieser Studie wurden mehr als 550 Zuweisungen in eine stationäre allgemeinpsychiatrische Behandlung evaluiert. Die Evaluation von Wirtz und Fromberger (2013) ergab eine „deutliche Diskrepanz in der diagnostischen Praxis“"zwischen den zuweisenden Fachärzten (für Psychiatrie und Psychotherapie sowie psychotherapeutische und psychosomatische Medizin) und den Diagnosen, welche mittels strukturierter diagnostischer Einschätzung (Screening auf Dissoziation, bei positiven Ergebnissen weitere Abklärung mittels strukturiertem klinischem Interview, SCID-D) durch die Kliniker erhoben wurden.

Weiters kann beispielsweise die Abgrenzung der Diagnose PTBS u. a. von folgenden assoziierten Diagnosen erforderlich sein: akute Belastungsreaktion, Angststörungen, komplexe Trauma-Folgestörung, Anpassungsstörung, komplizierte Trauer (Köllner und Maercker 2011). Nach Angaben der Leitlinie S3 Posttraumatische Belastungsstörung (Flatten et al. 2011) zählen zu den PTBS verwandten Störungsbildern: die akute Belastungsreaktion, die Anpassungsstörung, sowie die andauernde Persönlichkeitsänderung nach Extrembelastung. Dissoziative Störungsbilder, somatoforme Schmerzstörung und emotional instabile Persönlichkeitsstörung können ebenfalls als Trauma-Folgestörungen verstanden werden. Weitere Störungen, bei denen traumatische Belastungen laut Leitlinie „maßgeblich mitbedingend“ sind, sind die dissoziale Persönlichkeitsstörung, affektive Stö-

\footnotetext{
5 Gesehen 16.01.2017, http://oent.at/therapeutinnen.

${ }^{6}$ Gesehen 16.01.2017, http://www.emdr-netzwerk.at/ therapeutinnen.html
} 
rungen, Substanzabhängigkeit, Essstörungen sowie somatoforme Störungen (Flatten et al. 2011).

Abgesehen davon wird eine (Differenzial-) Diagnose oft erst im Laufe der Therapie möglich - weil Informationen nur vertrauten TherapeutInnen gegeben oder erst durch therapeutische Interventionen zugänglich werden. Diagnostische Interventionen sind immer auch therapeutisch relevant - in der psychotherapeutischen (wie auch in der traumapädagpogischen und psychosozialen) Arbeit mit komplex Traumatisierten zieht sich der theragnostische Prozess oft über mehrere Monate oder auch Jahre (Petzold 1974; Gahleitner und Rothdeutsch-Granzer 2016), bis unter der präsentierten Symptomatik liegende Traumata sicht-, erzähl- und behandelbar werden.

Es ergeben sich große Herausforderungen in der therapeutischen Arbeit, wenn die Diagnose erst im Laufe der Zeit „komplexe Traumatisierung mit Dissoziation“, vielleicht sogar deutlich ausgeprägte strukturelle Dissoziation, lautet. Kann/soll eine KollegIn an jemanden mit traumaspezifischer Zusatz-Qualifikation überweisen, auch wenn der Beziehungsaufbau mit diesem Klienten/dieser KlientIn gut begonnen hat? Unter anderem zu dieser Herausforderung hat auch die anerkannte deutsche Psychotraumatologin Michaela Huber in ihrem Interview in dieser Ausgabe des psychotherapie forum Stellung genommen, ihre Empfehlungen können im Interview nachgelesen werden.

Für diesen Prozess der Diagnosefindung müsste finanzierte Zeit zur Verfügung stehen (siehe Forderungen weiter unten im Text). Traumatherapeutische und diagnostische Ausbildungen sollten sich an den Richtlinien der DeGPT orientieren (siehe weiter oben im Text), Fachspezifika ein Basiswissen zu Trauma bereitstellen (siehe Forderungen weiter unten im Text). Eine oft entlastende Möglichkeit für die freie Praxis, die in der Regel bei längeren kassen-finanzierten Therapien zwingend vorgegeben ist, ist die Überweisung zur Psychodiagnostik.

\section{Trauma-sensibler Umgang im professionellen Umfeld}

Immer wieder berichten KlientInnen von entwürdigenden Erfahrungen durch Ungeduld, Kritik, mangelndem Verständnis im Rahmen von Kontakten mit Helfersystemen. Diagnostik-Termine oder Kontrollen im Rahmen von Kassenbegutachtungen führen bisweilen zu Irritation, gar Krisen. Muttersprachliche Angebote stehen kaum zur Verfügung und webbasierte Angebote sind erst im Kommen (Wagner 2016). Dissoziation wird teilweise immer noch als PhantasieProdukt bzw. von TherapeutInnen Eingeredetes abgewertet - der Forscher und Kliniker Ellert Nijenhuis argumentiert dagegen sowohl historisch-diskursiv als auch wissenschaftlich-analysierend in seinem letzten Buch „Die Trauma-Trinität“ (Nijenhuis 2016). Span- nend dazu auch die unterschiedlichen Artikel im letzten Psychotherapieforum (2016).

KlientInnen berichten, auch manche Reha-KlinikMitarbeitende sind auf komplex traumatisierte PatientInnen nicht optimal vorbereitet, obwohl mit „Trauma“ als Zuweisungsdiagnose auf der Website der Institution geworben wird. Traumatisierte KlientInnen reagieren im neuen Umfeld möglicherweise emotional instabil und verteidigend oder mit Verschlechterung anderer Symptome. Dies kann als Widerstand gewertet werden - die KlientInnen werden unter Umständen entwertet, vorzeitig entlassen, nach Hause geschickt. In der laufenden Therapie müssen daraus folgend häufig enttäuschte Erwartungen, Versagens- und Scham-Gefühle, Vertrauensverluste ins medizinische System bearbeitet werden. Oft sind während eines Reha-Aufenthaltes telefonische Helferkontakte hilfreich, in denen psycho-edukativ Verständnis hergestellt werden kann.

\section{Krisenmanagement}

Krisen, auch suizidale, sind bei komplex traumatisierten KlientInnen nicht selten. Sie können rasch und unvorhersehbar, durch das plötzliche Wiedererleben von Aspekten einer Trauma-Erfahrung oder Re-Traumatisierungen, ausgelöst werden.

Eine Einheit Psychotherapie pro Woche reicht oft nicht aus, um Halt zu finden - mehrere Einheiten sind aber nicht oder kaum finanzierbar bzw. werden vom Gesundheitssystem nicht gefördert - auch Krankenkassenzuschüsse sind streng limitiert. Einige KlientInnen suchen daher nach Kontaktmöglichkeiten zwischen den Einheiten per SMS, WhatsApp, Email, Anrufen. Aus Sicht der KlientInnen ist dies durchaus sinnvoll im Sinne der Selbstfürsorge in Krisenzeiten. TherapeutInnen müssen aber immer wieder abwägen, wie viel hier neben den regulären Therapiestunden noch geleistet werden kann und ob bzw. in welchem Maße diese zusätzlichen Leistungen verrechnet werden. Natürlich haben all diese Faktoren auch einen Einfluss auf die therapeutische Beziehung und das Selbstmanagement der KlientInnen. Bei web-basierter Kommunikation ist weiters auf den Datenschutz zu achten - siehe Internet-Richtlinie für Psychotherapeutinnen und Psychotherapeuten des Bundesministeriums für Gesundheit (Bundesministerium für $\mathrm{Ge}$ sundheit, 2005).

\section{Beziehungsgestaltung zwischen Ambivalenzen}

Die Beziehungsgestaltung mit traumatisierten KlientInnen erfordert je nach Stärke der Traumadynamik einiges an „Fingerspitzengefühl“. Bindungstraumatisierungen (vgl. Brisch 2017) in früher Kindheit haben häufig massive Folgen für die Beziehungsgestaltung auch im „Alltag“ der therapeutischen Beziehung (Stichwort „Übertragung“) Häufig besteht der Wunsch, Verantwortung für das eigene Leben weitge- 
hend abzugeben, der sich auch in der Therapie zeigt. Grenzziehungen von TherapeutInnen werden in diesem Spannungsfeld manchmal sehr kränkend erlebt und können Abbrüche nach sich ziehen. Supervisorische/intervisorische Reflexion, die eine wertschätzende Grenzziehung ohne Ärger unterstützt, und eine realistische, eher unterschätzende Erwartungshaltung seitens der TherapeutInnen sind hilfreich, damit Abgrenzung in Kontakt gelingen kann.

\section{Finanzierung}

Generell sind komplex traumatisierte KlientInnen häufig nicht in der Lage, in vollem Ausmaß erwerbstätig zu sein bzw. üben aufgrund so mancher Einschränkung Tätigkeiten aus, die nicht ihren Qualifikationen entsprechen und geringer bezahlt werden. Geld ist dann knapp und die Teilfinanzierung eines Therapieplatzes, die ja für die KlientInnen meist noch $75 \%$ (oder auch mehr) Selbstbehalt bedeutet, schwierig.

Dies bringt nicht nur die KlientInnen unter Druck, sondern auch TherapeutInnen. Stabilisierung und Ressourcenaufbau sowie auch Theragnose (Petzold 1974) als wichtige Bausteine Trauma-therapeutischen Arbeitens benötigen oft viele Sitzungen. Vor Beginn Trauma-aufdeckenden Arbeitens sollte geklärt sein, ob die/der KlientIn sich die nächsten Monate, vielleicht Jahre kontinuierlicher Therapie leisten kann bei etwaiger Stundenerhöhung in Krisen, denn unfreiwillige Therapieabbrüche werden sehr häufig als Re-Traumatisierung erlebt.

Eine jüngst veröffentlichte Studie zur psychotherapeutischen Versorgungsrealität komplex traumatisierter Menschen in Deutschland ergibt hierzu alarmierende Ergebnisse: als Auswirkungen einer längeren unfreiwilligen Unterbrechung der (voll kassenfinanzierten) ambulanten Psychotherapie aufgrund der Kassenfinanzierungsvorgaben gaben $74,5 \%$ der Betroffenen an, die Unterbrechung für den Heilungsprozess nicht hilfreich erlebt zu haben. 80,1\% gaben Behinderungen im therapeutischen Prozess an oder erlebten sich in diesem zurückgeworfen. Mehr als zwei Drittel der Befragten (68,5\%) gaben einen Vertrauensverlust in die therapeutische Beziehung an. $82,6 \%$ beobachteten verstärkt somatische Symptome oder neue. Es wurde erhöhter Bedarf an medikamentöser Behandlung bei $54,1 \%$ sowie eine Zunahme von selbstverletzendem Verhalten bei $61,8 \%$ erhoben. Eine Zunahme von suizidalen Tendenzen wurde von $68,5 \%$ berichtet; $46,5 \%$ wurden stationär aufgenommen aufgrund der Unterbrechung der Therapie.

Wenn Geld knapp ist oder wird, wäre eine Weiterführung der Therapie besonders wichtig. Die Anzahl von fundiert traumatherapeutisch weitergebildeten PsychotherapeutInnen mit Kassenplätzen ist jedoch extrem gering. Das Jahres-Stundenkontingent für die Kassen-TherapeutInnen ist zudem limitiert, was kaum Flexibilität für zusätzliche Krisen-Sitzungen bietet. Die üblicherweise maximal teilrefundierte Anzahl von ca. 160 Sitzungen pro Klientin reicht oft nicht für die erforderliche Therapiedauer. Daher bieten viele KollegInnen Langzeit-Traumatherapie zu Sozialbzw. Nulltarif an. Dies hat Nachteile wie: sich als KlientIn „zu Dank verpflichtet“ fühlen, Beschämung, schlechtes Gewissen und den Druck, in der Therapie besonders „gut sein“ zu müssen, und, dass TherapeutInnen sich durch finanzielle Sonderregelungen „teilweise bis sehr finanziell belastet" fühlen (Sommer 2016).

Zusätzlich gibt es methodische Anforderungen an das Setting. Gerade bei Trauma-konfrontativen Verfahren wie z. B. EMDR sind oft Doppeleinheiten sinnvoll. Dies wird von manchen Kassen ermöglicht, von anderen nicht. Weitere Unterschiede der Anzahl antragsfreier Sitzungen, der Antragsformulare, der Höhe der Refundierung und deren Erlangen erschweren den Überblick und das Management für KlientInnen wie TherapeutInnen.

KlientInnen ist diese Administration oft kaum möglich. Kontakte mit Kassen, Behörden, etc. bieten großes Konfliktpotenzial. Unbezahlte engagierte Unterstützung wie z. B. die Abklärung der jeweiligen Kassenvorgaben für weitere Bewilligungen oder psychotherapeutische Stellungnahmen als Begleitschreiben für Begutachtungen können manches abfedern, kosten jedoch enorm Zeit.

\section{Interdisziplinarität}

Vernetzung mit verschiedenen Institutionen bzw. Personen ist zur optimalen Unterstützung auch außerhalb der Therapie notwendig bzw. sinnvoll. Je nach Hintergrund können beispielsweise die Gleichbehandlungsanwaltschaft bei Mobbingerfahrungen, der Frauennotruf oder Beratungsstellen v. a. bei Opfern von (akuten) Übergriffserfahrungen hilfreich sein. Bei Gerichtsverfahren und Zeugenschaft als Opfer von kriminellen Handlungen ist rasche Vernetzung mit diesen Stellen für KlientInnen und TherapeutInnen unumgänglich, um die KlientInnen optimal unterstützt durch schwierige Befragungen, Gerichtsverhandlungen und Schadensfolgebegutachtungen zu begleiten und dadurch Trauma-bedingter Dekompensation oder Retraumatisierung in diesen Stresssituationen vorzubeugen.

Auch psychotherapeutische/trauma-therapeutische Stellungnahmen sind hilfreich, um z. B. Gerichte, AMS, Reha-Einrichtungen etc. über Trauma und traumabedingte Folgen aufzuklären.

Wir möchten alle KollegInnen ermutigen, diese Unterstützungsarbeit für ihre KlientInnen zu leisten. $\mathrm{Zu}$ oft haben Menschen in der Vergangenheit nicht oder zu wenig unterstützt. Hier bietet sich in der Therapie Raum für effektive Bindungsarbeit über konkretes Tun im Sinne der KlientInnen.

Gleichzeitig kommen TherapeutInnen hier oft an die Grenzen ihrer Verantwortlichkeiten und Möglichkeiten. Bereitschaft wie Kapazität für diese zusätzli- 
chen (meist unbezahlten) Leistungen sind inter- wie intrapersonell unterschiedlich.

\section{Forderungen}

\section{Politik}

Politik und ExpertInnen des Gesundheitssystems sind gefragt, um Veränderungen unseres bestehenden Versorgungssystems für komplex traumatisierte KlientInnen zu entwickeln. Es braucht sowohl Verbesserungen in der Therapiefinanzierung (Ausweitung der maximalen Gesamtanzahl teil-/voll-finanzierter Einheiten, Ausweitung der Einheiten für voll-finanzierte Therapie) als auch in der Versorgung der KlientInnen in Krisenzeiten (teilstationäre Angebote, mehr Trauma-spezialisierte Versorgungseinrichtungen und Klinikbetten) bei gleichzeitiger Schonung der Behandelnden (Kassen-Vergütungssätze, Gesamtvertrag). Ein Teil-Krankenstand würde gesundheitsfördernde Aspekte der Arbeit leichter zugänglich machen, ohne zu überfordern.

\section{Ausbildung}

Es braucht eine Berücksichtigung der Forschungsergebnisse der letzten zwei Jahrzehnte zu Trauma, Traumafolgen und Traumabehandlung in den Fachspezifika! Nur durch Vermittlung eines fundierten Basiswissens bereits in den Fachspezifika kann erreicht werden, dass AbsolventInnen dieser Ausbildungen komplex traumatisierten Menschen fachlich fundiert begegnen oder sie ggf. auch gleich an spezialisierte KollegInnen weiter verweisen können.

Es wäre auch zu diskutieren, eine entsprechend intensive curriculare Weiterbildung für Psychotraumatherapie als Zusatz zur Fachrichtung nach den Richtlinien des Bundesministeriums zu kennzeichnen, so wie es beispielsweise jetzt für die Kinder- und Jugendlichen-Psychotherapie erfolgt ist, und dadurch diese Kompetenz für KonsumentInnen auch klar erkennbar auszuweisen.

Klinische DiagnostikerInnen sollten ebenfalls eine vertiefte Ausbildung in Psycho-Traumatologie erhalten sowie den traumasensiblen Umgang mit Betroffenen einüben können.

\section{Anregungen für die Praxis}

\section{Supervision, Intervision, Fortbildung \& Psychohygiene}

Essentiell ist ein supportives kollegiales Umfeld. Intervision, Supervision, Fort- und Weiterbildung sind unverzichtbare Elemente. Eine Stunde Supervision bzw. Intervision pro Woche scheint nach Erfahrung der Autorinnen (als Minimum!) indiziert. Das erfordert Aufbau und Pflege eines entsprechenden Netzwerkes sowie Energie, Zeit und Geld. Diese Investition al- lerdings bietet nachhaltig einen wesentlichen Beitrag zu Qualitätssicherung und Psychohygiene. Austausch, Informationsweitergabe, Behandlungsplanung, Sichtbarmachen von Trauma-Dynamik etc. sollten hier ebenso Platz finden wie emotionale Unterstützung. (Dazu siehe auch das Interview mit Michaela Huber in diesem Heft.) Kontinuierliche Fort- und Weiterbildung mittels Fachliteratur und das Besuchen von Workshops sind ebenso qualitätssichernd wie Bestandteil von Selbstfürsorge, denn mit jedem Mehr an Know-how steigt die Arbeitszufriedenheit und das Wissen um die realistischen Möglichkeiten und Grenzen unserer Arbeit.

\section{Informationsmanagement}

Gerade in der Trauma-therapeutischen Praxis ist Dokumentation wesentlich. Einerseits gibt es oft komplexe Verrechnungsmodi, andererseits ist die Arbeit strukturiert und folgt einem klaren Behandlungskonzept. Es ist wichtig, auf Anhieb einen raschen Überblick über Problematiken und Ressourcen, schon Bearbeitetes und noch Offenes zu haben. Auch für die rasche Weitergabe von Information über ergänzende Unterstützungsangebote, fachliche Weiterempfehlungen (ÄrztInnen, andere Behandlungsmöglichkeiten) oder Diagnostikbögen sowie eigene Arbeitsmaterialien (Ressourcenlisten, Protokolle der durchgeführten Interventionen) empfiehlt sich Übersicht zur Erleichterung der praktischen Arbeit. Auch (aktuelle) Listen mit Vernetzungspartnern etc. sind hilfreich.

\section{Innere Haltung und Humor}

Die Haltung, dass Trauma-Folgesymptome die normale Reaktion normaler Menschen auf nicht normale Erlebnisse darstellen, ermöglicht einen wertschätzenden Umgang auf Augenhöhe. Der Einbezug der KlientInnen in alle Überlegungen und Entscheidungen trägt dazu bei, eine zunehmend tragfähige Arbeitsbeziehung mit klaren Grenzen und Verantwortlichkeiten zu etablieren, die unterstützt und weder überfordert noch entmündigt.

Und wenn möglich, zwischendurch lachen mit KlientInnen! Humor ist auch in der Traumatherapie erlaubt und oft sehr hilfreich.

\section{Unsere top-10 Tipps für Trauma-TherapeutInnen}

1. regelmäßige Supervision mit erfahrenen ExpertInnen (Lernen aus Wissen und Erfahrung)

2. engmaschige kollegiale Reflexion der eigenen Arbeit in einem offenen Klima (Intervision)

3. regelmäßige Fortbildung, um up-to-date zu sein

4. immer wieder neuere Fachliteratur lesen

5. Vernetzung mit anderen Berufsgruppen und mit KollegInnen pflegen

6. Diagnostik, Behandlungsplanung, Beziehungsdynamik gleichermaßen beachten 
7. Bewusstheit über eigene Grenzen entwickeln und auf Wahrung dieser achten

8. das eigene Wohlbefinden wichtig nehmen und für eigene Balance sorgen

9. übersichtliches Informationsmanagement in der Praxis anstreben

10. mit Humor und Mut KlientInnen auf Augenhöhe begegnen

Interessenkonflikt M. Bernhaupt-Hopfner und T. Kosicek geben an, dass kein Interessenkonflikt besteht.

Open Access Dieser Artikel wird unter der Creative Commons Namensnennung 4.0 International Lizenz (http:// creativecommons.org/licenses/by/4.0/deed.de) veröffentlicht, welche die Nutzung, Vervielfältigung, Bearbeitung, Verbreitung und Wiedergabe in jeglichem Medium und Format erlaubt, sofern Sie den/die ursprünglichen Autor(en) und die Quelle ordnungsgemäß nennen, einen Link zur Creative Commons Lizenz beifügen und angeben, ob Änderungen vorgenommen wurden.

\section{Literatur}

Brisch, K.-H. (2017). Bindungstraumatisierungen. Wenn Bindungspersonen zu Tätern werden. Stuttgart: Klett-Cotta.

Bundesministerium für Gesundheit (2005). Internetrichtlinie für Psychotherapeutinnen und Psychotherapeuten. http://www.bmgf.gv.at/cms/home/attachments/ 7/0/5/CH1002/CMS1415709133783/internetrichtlinie_ 03052012.pdf.Zugegriffen: 16. Jan 2017.

Flatten, G., Gast, U., Hofmann, A., Knaevelsrud, C., Lampe, A., Liebermann, P., Maercker, A., Reddemann, L., \&Wöller, W. (2011). S3-Leitlinie Posttraumatische Belastungsstörung ICD-10:F43.1. Trauma \& Gewalt, 5, 202-211.

Gahleitner, S. B. (2010). Das „Therapeutische Milieu“ als Antwort auffrühe Gewalterfahrung. DerPersonzentrierteAn- satz bei komplexer Traumatisierung. Trauma \& Gewalt, $4(02), 129-140$.

Gahleitner, S. B., \& Rothdeutsch-Granzer, C. (2016). „Traumatherapie, Traumaberatung, Traumapädagogik - ein aktueller Überblick über neue Interventionsformen bei komplexen Traumafolgestörungen" Gemeinsamkeiten und Unterschiede zwischen verschiedenen Behandlungsformen. Psychotherapieforum, 4, 142-148.

Herman, J. (1992). Complex PTB: a syndrome in survivers of prolonged and repeated trauma. Journal of Traumatic Stress, 5, 377-391.

Köllner, V., \& Maercker, A. (2011). Das diagnostische Spektrum derTraumafolgestörungen. Trauma \& Gewalt, 5(03), 236-247.

Nijenhuis, E. (2016). Die Trauma-Trinität. Ignoranz - Fragilität - Kontrolle: Die Entwicklung des Traumabegriffs/ Theorie und Praxis traumabedingter Dissoziation. Göttingen: Vandenhoeck \& Ruprecht.

Petzold, H. G. (1974). Die diagnostischen und therapeutischen Möglichkeiten des Psychodramas im „tetradischen System“. Dynamische Psychiatrie, 3, 151-181.

Sack, M. (2001). Interview zur komplexen Posttraumatischen Belastungsstörung (IK-PTBS). Erstellt auf der Grundlage des SIDES-Interviews. Autoren: Van der Kolk, Pelcovitz, Herman, Roth, Kaplan, Waldinger, Guastela, Spitzer (1999); dt. Übersetzung und Bearbeitung: Sack, Hofmann (2001). http://www.martinsack.de/_downloads/ Interview_zur_komplexen_PTBS.pdf.Zugegriffen:16.Jan 2017.

Sommer, J. (2016). Die psychotherapeutische Versorgungsrealität komplex traumatisierter Menschen in Deutschland. Trauma \& Gewalt, 10(04), 308-319.

Wagner, B. (2016). Online-Therapie - eine neue Perspektive in der Psychotherapie für Flüchtlinge und Asylbewerber? Psychotherapieforum, 4, 124-131.

Weltgesundheitsorganisation WHO (1994). Internationale Klassifikation psychischer Störungen. ICD-10 Kapitel V (F). Bern:Huber.

Wirtz, G., \& Fromberger, U. (2013). Diagnostik dissoziativer Störungen in der ambulanten und stationären Behandlung. Trauma \& Gewalt, 7(03), 182-191. 